\title{
Possible depletion of metals into dust grains in the core of the Centaurus cluster of galaxies
}

\author{
K. Lakhchaura ${ }^{1,2}$, F. Mernier ${ }^{1,3}$, and N. Werner ${ }^{1,4,5}$ \\ 1 MTA-Eötvös University Lendület Hot Universe Research Group, Pázmány Péter sétány 1/A, Budapest 1117, Hungary \\ e-mail: lakhchaura.k@gmail.com \\ 2 MTA-ELTE Astrophysics Research Group, Pázmány Péter sétány 1/A, Budapest 1117, Hungary \\ 3 SRON Netherlands Institute for Space Research, Sorbonnelaan 2, 3584 CA Utrecht, The Netherlands \\ 4 Department of Theoretical Physics and Astrophysics, Faculty of Science, Masaryk University, Kotlářská 2, \\ Brno 611 37, Czech Republic \\ 5 School of Science, Hiroshima University, 1-3-1 Kagamiyama, Higashi-Hiroshima 739-8526, Japan
}

Received 30 November 2018 / Accepted 12 December 2018

\begin{abstract}
We present azimuthally averaged metal abundance profiles from a full, comprehensive, and conservative re-analysis of the deep ( $~ 800 \mathrm{ks}$ total net exposure) Chandra/ACIS-S observation of the Centaurus cluster core (NGC 4696). After carefully checking various sources of systematic uncertainties, including the choice of the spectral deprojection method, assumptions about the temperature structure of the gas, and uncertainties in the continuum modeling, we confirm the existence of a central drop in the abundances of the "reactive" elements $\mathrm{Fe}, \mathrm{Si}, \mathrm{S}, \mathrm{Mg}$, and $\mathrm{Ca}$, within $r \lessgtr 10 \mathrm{kpc}$. The same drops are also found when analyzing the XMM-Newton/EPIC data ( $\sim 150 \mathrm{ks})$. Adopting our most conservative approach, we find that, unlike the central drops seen for Fe, $\mathrm{Si}, \mathrm{S}, \mathrm{Mg}$ and $\mathrm{Ca}$, the abundance of the "nonreactive" element Ar is fully consistent with showing no central drop. This is further confirmed by the significant $(>3 \sigma)$ central radial increase of the Ar/Fe ratio. Our results corroborate the previously proposed "dust depletion scenario", in which central metal abundance drops are explained by the deposition of a significant fraction of centrally cooled reactive metals into dust grains present in the central regions of the Centaurus cluster. This is also supported by the previous findings that the extent of the metal abundance drops in NGC 4696 broadly coincides with the infrared dust emission.
\end{abstract}

Key words. galaxies: abundances - galaxies: evolution - galaxies: active - X-rays: galaxies: clusters

\section{Introduction}

One of the most compelling ways to study the chemical evolution of the universe at its largest scales is via X-ray spectroscopy of galaxy clusters, groups, and giant elliptical galaxies. Indeed, the gravitational potential wells of these systems are deep enough to retain the major fraction of their baryons, which consist of a hot $\left(10^{6}-10^{8} \mathrm{~K}\right)$, tenuous $\left(10^{-1}-10^{-5} \mathrm{~cm}^{-3}\right) \mathrm{X}$-rayemitting plasma (for a review, see e.g., Böhringer \& Werner 2010). These hot atmospheres are rich in heavy elements $(\mathrm{C}$, $\mathrm{N}, \mathrm{O}, \mathrm{Ne}, \mathrm{Mg}, \mathrm{Si}, \mathrm{S}, \mathrm{Ar}, \mathrm{Ca}, \mathrm{Cr}, \mathrm{Mn}, \mathrm{Fe}$, and Ni) whose abundances can be relatively well constrained from their $\mathrm{K}$-shell emission lines in the X-ray energy window (for a recent review, see Mernier et al. 2018a).

Thanks to current X-ray observatories like Chandra and $X M M-N e w t o n$, which allow to do accurate spatial spectroscopy, the spatial distribution of metals in the diffuse intracluster medium (ICM) can be studied in detail, providing invaluable information on where, how, and when these metals - produced by Type Ia (SNIa) and core-collapse supernovae (SNcc) escaped from their galaxy hosts and enriched hot atmospheres of galaxy clusters, groups, and ellipticals. In the outskirts, the recent discovery of flat, uniform metal abundance profiles (Werner et al. 2013; Simionescu et al. 2015; Urban et al. 2017) strongly suggests that the bulk of metals enriched the hot ICM before clusters started to form and attain a well stratified entropy (i.e., typically at $z \gtrsim 2-3$ ). This scenario is also supported by recent cosmological hydrodynamical simulations (Biffi et al.
2017, 2018a; Truong et al. 2019), suggesting that active galactic nuclei (AGNs) play an essential role in spreading metals out of their host galaxies before assembling into clusters (for a review, see e.g., Biffi et al. 2018b). In addition, it is now well established that cool-core clusters ${ }^{1}$ host a centrally peaked $\mathrm{Fe}$ distribution while noncool-core clusters exhibit a much flatter Fe profile (De Grandi et al. 2004; Lovisari \& Reiprich 2019). The central Fe excess found in cool-core clusters was initially thought to be produced by ongoing SNIa explosions from the low-mass stellar population within the (red-and-dead) brightest cluster galaxies (BCG; e.g., Böhringer et al. 2004). However, the surprising finding that SNcc products (i.e., $\mathrm{O}, \mathrm{Mg}$, as well as a fraction of $\mathrm{Si}, \mathrm{S}$, and $\mathrm{Ar}$ ), which are not expected to be produced by BCGs within the last $\sim 7-8 \mathrm{Gyr}$, also exhibit a central peak reopened the question of the origin of the central enrichment in cool-core systems (Simionescu et al. 2009; Mernier et al. 2017).

Perhaps even more intriguing is the presence of apparent metal abundance drops in the very core (i.e., within a few kiloparsecs) of some relaxed systems. First discovered in the galaxy cluster Abell 2199 (Johnstone et al. 2002), central drops were later reported for Fe in many other sources, including also less massive groups and ellipticals (e.g., Churazov et al. 2003, 2004; Rasmussen \& Ponman 2007; Rafferty et al. 2013; Panagoulia et al. 2015; Mernier et al. 2017;

\footnotetext{
1 The term "cool-core" is used for systems in which the cooling time of the central ICM is typically shorter than the Hubble time (Molendi \& Pizzolato 2001; Hudson et al. 2010).
} 
Gendron-Marsolais et al. 2017). Because these drops are difficult to explain with simple enrichment models, they were initially thought to be artefacts of spectral analysis. For instance, Werner et al. (2006) showed that the apparent drop seen in 2A 0335+096 was solely due to the oversimplified assumption of a single-temperature plasma, as no inversion in the Fe profile is found when two or more temperatures are modeled in the spectra (i.e., the so-called "Fe-bias"; e.g., Buote 2000). In several other cases, however, assuming a multi-temperature structure for the gas does not help to attenuate these drops. Possible effects from resonance scattering have also been proposed, but were found to contribute to only a negligible fraction of the apparently missing Fe (Sanders \& Fabian 2006b; Gendron-Marsolais et al. 2017). Besides Fe, central abundance drops were also found for several other elements, in particular Si and S (Panagoulia et al. 2015; Mernier et al. 2017).

Under the assumption that the observed metal abundance drops are real, it has been proposed (Panagoulia et al. 2013, 2015) that a significant fraction of metals initially residing in the hot phase will rapidly cool down and be deposited into dust grains, thereby becoming invisible in the X-ray band. As most BCGs host an AGN which is able to uplift and re-heat its surrounding hot atmosphere via mechanical feedback (for a recent review, see Werner et al. 2019), metal-rich dust grains may be dragged out of the innermost regions, possibly in the form of extended emission line filaments, before becoming re-heated and returning into their initial hot phase a few kiloparsecs away from the center.

A key prediction of the above scenario is that, unlike all the metal abundances that are measurable in X-ray (i.e., "reactive" elements), abundance drops are not expected to occur for $\mathrm{Ne}$ and Ar, as these two elements are noble gases and are not easily deposited into dust grains (i.e., "non-reactive"). The current CCD instruments do not have the required spectral resolution to accurately constrain the $\mathrm{Ne}$ abundance ${ }^{2}$ and no firm conclusion could be drawn from the central Ar profiles using XMM-Newton observations of nearby cool-core systems (Mernier et al. 2017). In addition to the moderate spatial resolution of XMM-Newton, the main reason is that stacking data of many systems naturally increases the intrinsic scatter of the profiles, especially in coolcore systems (see also Lovisari \& Reiprich 2019). Clearly, testing the above scenario requires very deep observations of one bright, carefully selected, nearby cool-core system.

The most striking example of central metal abundance drops certainly comes from Abell 3526 (i.e., the Centaurus cluster). The central BCG (NGC 4696) of this bright and nearby coolcore system exhibits one of the most prominent Fe drops known to date (with an abrupt inwards decrease from $\sim 2$ to $\sim 0.5$ solar), which was the second ever discovered (Sanders \& Fabian 2002). Since then, additional detailed observations of the Centaurus cluster have further confirmed this remarkable drop within its central 5-20 kpc, not only in $\mathrm{Fe}$ but also in $\mathrm{Si}, \mathrm{S}$, and possibly Mg (Sanders \& Fabian 2006a; Panagoulia et al. 2013; Sanders et al. 2016). In addition, NGC 4696 is well known to contain significant amounts of dust, observed in far-infrared with Spitzer (Kaneda et al. 2005, 2007) and Herschel (Mittal et al. 2011), as well as extended $\mathrm{H} \alpha$ and optical (in particular

\footnotetext{
2 Unfortunately, the main $\mathrm{Ne}$ emission lines ( $\mathrm{Nex}$ and $\mathrm{NexI}$ ) are located within the Fe-L complex unresolved by CCD instruments, making the $\mathrm{Ne}$ abundance strongly degenerate with other parameters, e.g., the $\mathrm{Fe}$ abundance or the temperature structure of the gas. While the Reflection Grating Spectrometer (RGS) instrument on-board $X M M-N e w t o n$ is technically able to resolve these lines, its slitless design does not allow for spatially resolved spectroscopy.
}

[NII]) filaments (Fabian et al. 2016; Hamer et al. 2019, see also Lakhchaura et al. 2018).

In this paper, we take advantage of the extremely deep Chandra observations of NGC 4696 (residing in the core of the Centaurus cluster), obtained in successive pointings over 14 years since the beginning of the mission, combined with the unique spatial resolution of the telescope, in order to infer unprecedented constraints on its metal abundance profiles. Specifically, we aim to compare the (non-reactive) Ar radial distribution with that of the other (reactive) elements in order to test the dust depletion scenario as a possible explanation of the central metal abundance drops. In this respect, we put a strong emphasis on the major systematic effects that may alter such measurements and possibly explain some results previously reported in the literature. This includes a careful comparison with XMM-Newton measurements, which we also re-analyze. The data reduction and analysis of all the Chandra and XMMNewton observations are described in Sect. 2. Our results are reported in Sect. 3, and are subsequently discussed and further interpreted in Sect. 4. We summarize our conclusions in Sect. 5. Throughout this paper, we adopt a $\Lambda$ CDM cosmology with $H_{0}=70 \mathrm{~km} \mathrm{~s}^{-1} \mathrm{Mpc}^{-1}, \Omega_{\mathrm{M}}=0.3$, and $\Omega_{\Lambda}=0.7$. The metal abundances are given with respect to the solar values of Grevesse \& Sauval (1998). Unless stated otherwise, the errors are given within their $68.27 \%$ confidence level $(1 \sigma)$.

\section{Data}

For this work, we used all the publicly available Chandra and XMM-Newton observations of the core of the Centaurus cluster (NGC 4696), taken from the High-Energy Astrophysics Science Archive Research Center (HEASARC). A log of these observations is given in Table 1.

\subsection{Chandra data reduction and analysis}

We used the Chandra Interactive Analysis of Observations (CIAO) software version 4.9 (Fruscione et al. 2006) and CALDB version 4.7.3 for all the data reduction, and for the spectral analyses, we used the X-ray spectral fitting package XSPEC version 12.9.1 (Arnaud 1996). The procedures used for the data reduction were the same as described in Lakhchaura et al. (2018). The standard chandra_repro tool was used to reprocess the data and strong background flares were filtered and removed using the 1c_clean script. Once filtered, the cleaned ACIS-S data have a total exposure of $797.31 \mathrm{ks}$ (Table 1). The point sources in the field were detected using the CIAO task wavdetect (false-positive probability threshold $=10^{-6}$ ), verified by visual inspection of the X-ray images and removed from the subsequent analysis.

\subsubsection{Spectral extraction}

Although the remarkable amount of data available for NGC 4696 allows for the spectral regions to be subdivided into multiple, azimuthally resolved spatial bins (e.g., Sanders et al. 2016), here we aim to optimize the number of counts available per region in order to provide the most accurate constraints or the Ar abundance. This implies adopting radial bins that are both azimuthally averaged (i.e., concentric annuli instead of sectors) and much larger than what is usually considered based on the spatial resolution of Chandra. Therefore, spectra were extracted from four concentric annuli (or shells, when the spectra are further deprojected), bounded by 4.4, 11.0, 26.6, and $65.3 \mathrm{kpc}$ (corresponding to $\sim 0.4,1.0,2.5,6.0$ arcmin, respectively; see Fig. 1) 
Table 1. Log of the Chandra and XMM-Newton observations of NGC 4696, used in this paper.

\begin{tabular}{|c|c|c|c|}
\hline Obs ID & Instrument & $\begin{array}{c}\text { Cleaned exp. } \\
(\mathrm{ks})\end{array}$ & $\begin{array}{c}\text { Date of } \\
\text { observation }\end{array}$ \\
\hline \multicolumn{4}{|c|}{ Chandra } \\
\hline 504 & ACIS-S & 31.48 & $2000-05-22$ \\
\hline 505 & ACIS-S & 9.96 & 2000-06-08 \\
\hline 1560 & ACIS-S & 45.56 & 2001-04-18 \\
\hline 4190 & ACIS-S & 34.00 & 2003-04-18 \\
\hline 4191 & ACIS-S & 32.74 & 2003-04-18 \\
\hline 4954 & ACIS-S & 87.26 & 2004-04-01 \\
\hline 4955 & ACIS-S & 44.68 & 2004-04-02 \\
\hline 5310 & ACIS-S & 36.24 & 2004-04-04 \\
\hline 16223 & ACIS-S & 175.38 & $2014-05-26$ \\
\hline 16224 & ACIS-S & 40.76 & 2014-04-09 \\
\hline 16225 & ACIS-S & 29.33 & $2014-04-26$ \\
\hline 16534 & ACIS-S & 54.68 & 2014-06-05 \\
\hline 16607 & ACIS-S & 44.78 & 2014-04-12 \\
\hline 16608 & ACIS-S & 33.35 & 2014-04-07 \\
\hline 16609 & ACIS-S & 80.54 & 2014-05-04 \\
\hline 16610 & ACIS-S & 16.57 & 2014-04-27 \\
\hline \multicolumn{4}{|c|}{ XMM-Newton } \\
\hline 0046340101 & EPIC MOS 1 & 44.22 & 2002-01-03 \\
\hline 0046340101 & EPIC MOS 2 & 44.32 & 2002-01-03 \\
\hline 0046340101 & EPIC pn & 48.56 & $2002-01-03$ \\
\hline 0406200101 & EPIC MOS 1 & 107.64 & 2006-07-24 \\
\hline 0406200101 & EPIC MOS 2 & 108.81 & $2006-07-24$ \\
\hline 0406200101 & EPIC pn & 92.61 & 2006-07-24 \\
\hline
\end{tabular}

and centered on the X-ray emission peak, using the CIAO task specextract. The annuli selection lead to $\sim 0.76,1.51,2.61$ and 3.71 million counts in the four annuli, respectively, in the $0.5-7.0 \mathrm{keV}$ energy range.

The standard Chandra blank-sky background event files matching the source observations were used to extract the background spectrum corresponding to each of the source spectra. The blank-sky event files were reprojected to match the source observation coordinate frame. We also scaled all the blanksky spectra by the ratio of the $9.5-12 \mathrm{keV}$ count rates of the source and blank-sky observations, in order to match the timedependent particle background levels in the source and blanksky observations. The differences in the Galactic foreground level in the scaled blank-sky and source spectra, determined using the ROSAT All Sky Survey 0.47-1.21 keV (RASS 45 band) count rates, were found to be insignificant. We note that, whereas background issues can sometimes be appreciable when analyzing extended X-ray sources, the exceptional brightness of NGC 4696 makes background uncertainties largely negligible in the present analysis.

\subsubsection{Spectral fitting}

The gas in the central regions of Centaurus is known to be multiphase. Therefore, single-temperature plasma models cannot fit the spectra well enough in the innermost bins, and may even cause significant underestimation of Fe due to "Fe-bias" (Buote 2000, see also Mernier et al. 2018a). To account for the complex multi-temperature structure of the cluster core, we successively fitted the spectra with two different multi-temperature models, namely a two-component vapec model (using AtomDB version 3.0.7), hereafter "2T vapec", and a vgadem model, which reproduces a normally distributed differential emission measure. We also added an additional thermal bremsstrahlung component with the temperature fixed at $7.3 \mathrm{keV}$ to account for the unresolved low-mass X-ray binaries in NGC 4696 (see Irwin et al. 2003). The Galactic absorption was accounted for by using the photoelectric absorption model wabs. The neutral hydrogen column density and the redshift were kept as free parameters in all the analyses. We verified that, within a given annulus, a slight variation of these two best-fit parameters has negligible effects (i.e., a few percent at most) on our results.

For both the $2 \mathrm{~T}$ vapec and vgadem models, the $\mathrm{Fe}, \mathrm{Si}, \mathrm{S}$, $\mathrm{Mg}, \mathrm{Ca}$ and $\mathrm{Ar}$ abundances were kept as free parameters, the $\mathrm{He}$ abundance was fixed to the solar value, and all other elements were tied to Fe. For the 2T vapec model, the temperatures and normalizations of the two vapec components were kept as free parameters while the abundances of the same elements were tied between the two components. We also note that a singletemperature component (vapec) was used when modeling two components did not significantly improve the fits (which often results in highly degenerate parameters between the two components). For the vgadem model, the mean temperature, the width of the temperature distribution, and the normalization were kept as free parameters. To determine the gas density $\left(n=n_{\mathrm{e}}+n_{i}\right)$ of the four shells (assuming spherical symmetry), a constant density was assumed in each shell, and then the densities were obtained from the vapec/vgadem normalizations $(\eta)$ using the following relation.

$\eta=\frac{10^{-14} \int n_{\mathrm{e}} n_{\mathrm{p}} \mathrm{d} V}{4 \pi D_{\mathrm{A}}^{2}(1+\mathrm{z})^{2}}$,

where $D_{\mathrm{A}}$ is the angular diameter distance of the source, and $n_{\mathrm{e}}$ and $n_{\mathrm{p}}$ are the electron and proton number densities (for a fully ionized gas with one third solar elemental abundance $n_{\mathrm{e}}=0.53 n$ and $n_{\mathrm{p}}=n_{\mathrm{e}} / 1.2$ ), respectively.

\subsection{XMM-Newton data reduction and analysis}

We also compare the results obtained using the deep Chandra data with the archival XMM-Newton observations of the Centaurus core, which were taken in two separate pointings (January 2002 and July 2006). The data reduction was performed using the XMM-Newton Science Analysis System (SAS) v14.0.0 and follows the same procedure as presented in Mernier et al. (2015). In summary, we process the EPIC MOS (i.e., MOS 1 and MOS 2) and pn data using the tasks emproc and epproc, respectively, before filtering them to discard flared events. To do so, the light curves in the 10-12 keV band were stacked in $100 \mathrm{~s}$ binned histograms. We then fitted a Gaussian to the distribution and selected the count-rate threshold above which the events are rejected to be $\mu+2 \sigma$. The same procedure was repeated for the $0.3-2 \mathrm{keV}$ band (for $10 \mathrm{~s}$ binned histograms), as flaring events may affect softer energies differently (Lumb et al. 2002). A summary of the cleaned XMM-Newton data $(\sim 150 \mathrm{ks}$ for the two pointings) is also presented in Table 1.

The rest of the analysis of the XMM-Newton data follows that described above for Chandra (Sects. 2.1.1 and 2.1.2). Specifically, the EPIC spectra were extracted following the same regions as shown in Fig. 1 and the excluded point sources were selected from the ACIS-S detections. In order to account for the larger point spread function (PSF) of XMM-Newton, the circular regions centered on the point sources were excised within a radius of $10 \mathrm{arcsec}$ (Mernier et al. 2015). For the two XMM-Newton observations, the combined $0.5-7.0 \mathrm{keV}$ counts 

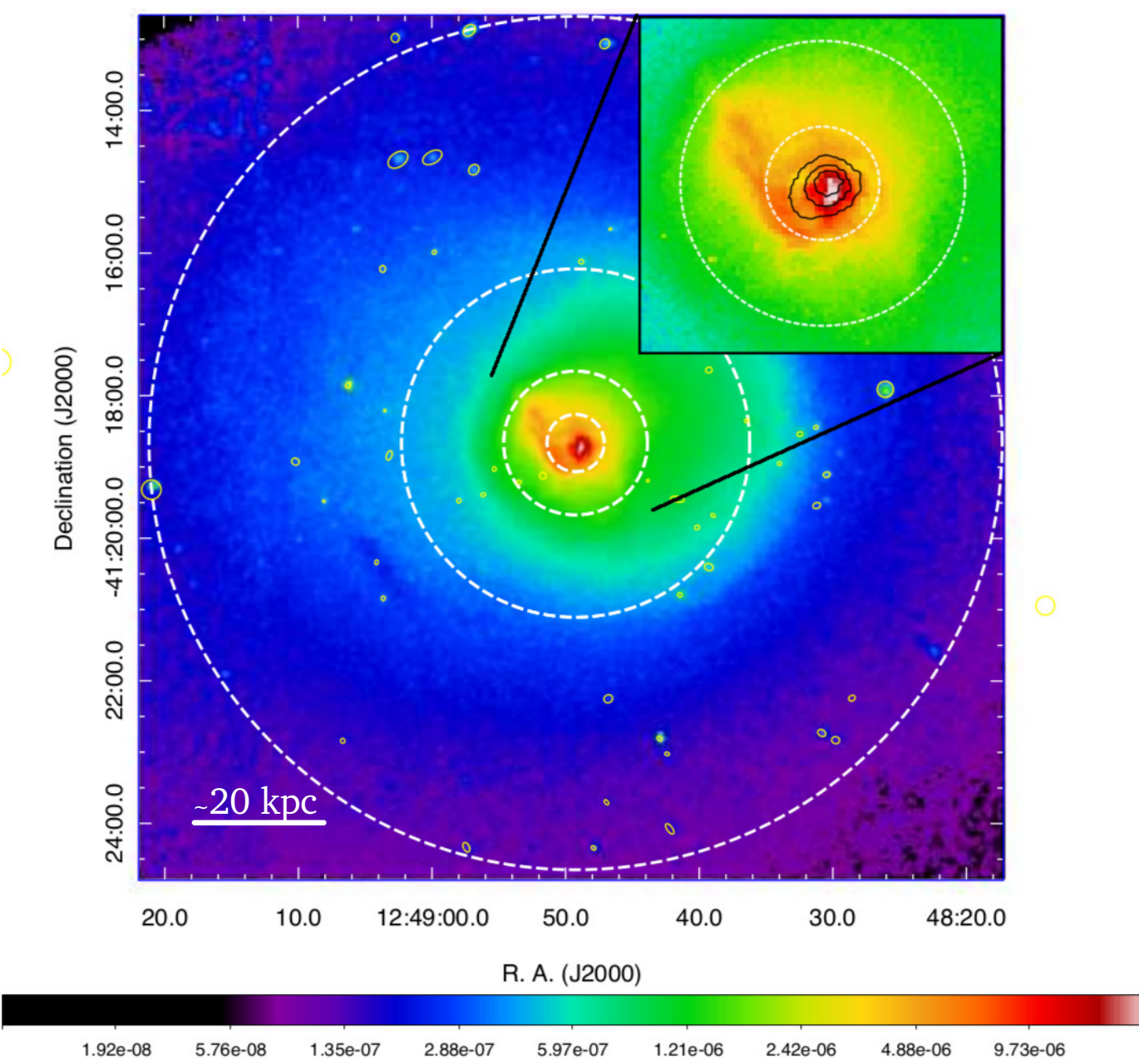

Fig. 1. An exposure corrected $0.5-7.0 \mathrm{keV}$ Chandra ACIS-S image of the Centaurus cluster. The color bar indicates the photon flux in counts $\mathrm{cm}^{-2} \mathrm{~s}^{-1}$. The white dashed circles show the four annular regions used for the spectral extraction. The inset is a zoomed-in section of the central region showing the central soft X-ray filaments and the asymmetric distribution of the central X-ray emission. The black contours in the inset are from a Herschel PACS $100 \mu \mathrm{m}$ photometric observation of the cluster, and trace the central dust emission.

in the four annuli were $\sim 0.11,0.25,0.45$ and 0.73 million for MOS 1; $0.11,0.24,0.46$ and 0.76 million for MOS 2; and $\sim 0.19,0.36,0.65$ and 1.00 million for pn, respectively. The RMFs and ARFs were obtained via the SAS tasks rmfgen and arfgen, respectively. The MOS 1, MOS 2, and pn spectra were all fitted simultaneously, with all their parameters tied accordingly between the three instruments.

\section{Results}

As stated in Sect. 1, we aim to carry out a comprehensive study on the central metal abundance drops in the Centaurus cluster, taking into account all the potential fitting biases that might significantly affect our measurements. In the following subsections, we compare our radial profiles obtained with different assumptions, namely the temperature structure of the gas, the choice of the spectral deprojection method, the energy band within which the spectra are fitted, and the instruments used.

\subsection{Projected profiles: "vgadem" versus " $2 T$ vapec" models}

First, we focus on the Chandra observations of NGC 4696 as directly seen by the ACIS-S instrument, that is, projected on the plane of the sky. As stated in Sect. 2.1.2, two types of multitemperature models are considered: vgadem and $2 \mathrm{~T}$ vapec. In the case of the $2 \mathrm{~T}$ vapec model, a single-temperature (vapec) component was enough to fit the spectra beyond the two innermost annuli. The projected profiles of temperature (i.e., the mean temperature for vgadem and the higher temperature for $2 \mathrm{~T}$ vapec), total number density, Fe, $\mathrm{Si}, \mathrm{S}, \mathrm{Mg}, \mathrm{Ca}$, and $\mathrm{Ar}$ abundances, and the $\mathrm{Ar} / \mathrm{Fe}$ ratio, obtained using these two models successively, are shown in Table 2 and Fig. 2 (red and blue filled areas).

Globally, these profiles seem to be very similar, and do not depend strongly on the choice of the multi-temperature model. We note, however, that the density is systematically higher in the 2 T vapec (by typically $\sim 30 \%$ ) than in the vgadem case. As expected, it clearly appears that, no matter which model is considered, the Fe, Si, S and $\mathrm{Mg}$ profiles show a central decrease at $r \lesssim 10 \mathrm{kpc}$. Drops are also reported for Ar and $\mathrm{Ca}$, although only at $r \lesssim 4 \mathrm{kpc}$. However, the $\mathrm{Ar} / \mathrm{Fe}$ ratio (Fig. 2, bottom right panel) seems to be increasing towards the center and the increase in the innermost bin, as compared to the outermost bin, is $>3 \sigma$ and $>2 \sigma$ when using the $2 \mathrm{~T}$ vapec and the vgadem models, respectively.

\subsection{Deprojected profiles}

Projection of X-ray emission along the line of sight can blur out actual variations in the temperature and metal-abundance distribution, and can sometimes also create artificial features. In order 
Table 2. Best-fit values for the temperature, density, $\mathrm{Fe}, \mathrm{Si}, \mathrm{S}, \mathrm{Mg}, \mathrm{Ca}$, and $\mathrm{Ar}$ abundances, and the $\mathrm{Ar} / \mathrm{Fe}$ ratio (for the narrow band analysis, the Fe abundance is taken from the broad-band fit), and their associated uncertainties (quoted at the $68.27 \%$ or $1 \sigma$ level) obtained from the various spectral fitting and deprojection methods employed in the paper.

\begin{tabular}{|c|c|c|c|c|c|c|c|c|c|}
\hline $\begin{array}{l}\text { Radial range } \\
(\mathrm{kpc})\end{array}$ & $\begin{array}{c}\mathrm{kT} \\
(\mathrm{keV})\end{array}$ & $\begin{array}{c}\mathrm{n} \\
\left(10^{-2} \mathrm{~cm}^{-3}\right)\end{array}$ & $\begin{array}{c}\mathrm{Fe} \\
\text { (solar) }\end{array}$ & $\begin{array}{c}\mathrm{Si} \\
\text { (solar) }\end{array}$ & $\begin{array}{c}\text { S } \\
\text { (solar) }\end{array}$ & $\begin{array}{l}\mathrm{Mg} \\
\text { (solar) }\end{array}$ & $\begin{array}{c}\mathrm{Ca} \\
\text { (solar) }\end{array}$ & $\begin{array}{c}\mathrm{Ar} \\
\text { (solar) }\end{array}$ & $\mathrm{Ar} / \mathrm{Fe}$ \\
\hline \multicolumn{10}{|c|}{ Chandra projected } \\
\hline \multicolumn{10}{|c|}{ Model: vgadem } \\
\hline $0-4.4$ & $1.600 \pm 0.002$ & $10.78 \pm 0.04$ & $1.53 \pm 0.01$ & $1.37 \pm 0.01$ & $1.05 \pm 0.01$ & $1.05 \pm 0.01$ & $1.53 \pm 0.06$ & $1.67 \pm 0.06$ & $1.09 \pm 0.04$ \\
\hline $4.4-11.0$ & $1.945 \pm 0.002$ & $4.02 \pm 0.01$ & $1.86 \pm 0.01$ & $1.79 \pm 0.01$ & $1.24 \pm 0.01$ & $1.07 \pm 0.01$ & $1.91 \pm 0.04$ & $2.10 \pm 0.04$ & $1.13 \pm 0.02$ \\
\hline $11.0-26.6$ & $2.648 \pm 0.003$ & $1.463 \pm 0.001$ & $1.90 \pm 0.01$ & $1.86 \pm 0.01$ & $1.24 \pm 0.01$ & $1.18 \pm 0.01$ & $1.64 \pm 0.03$ & $1.92 \pm 0.04$ & $1.01 \pm 0.02$ \\
\hline $26.6-65.3$ & $3.278 \pm 0.007$ & $0.4885 \pm 0.0004$ & $1.21 \pm 0.01$ & $1.23 \pm 0.01$ & $0.78 \pm 0.01$ & $1.10 \pm 0.01$ & $0.92 \pm 0.03$ & $1.08 \pm 0.04$ & $0.89 \pm 0.03$ \\
\hline \multicolumn{10}{|c|}{ Model: 2T vapec } \\
\hline $0-4.4$ & $1.604 \pm 0.003$ & $13.49 \pm 0.01$ & $1.406 \pm 0.005$ & $1.27 \pm 0.01$ & $0.98 \pm 0.01$ & $0.97 \pm 0.01$ & $1.62 \pm 0.06$ & $1.72 \pm 0.06$ & $1.22 \pm 0.04$ \\
\hline $4.4-11.0$ & $1.992 \pm 0.003$ & $5.428 \pm 0.004$ & $1.977 \pm 0.005$ & $1.77 \pm 0.01$ & $1.25 \pm 0.01$ & $1.07 \pm 0.01$ & $1.92 \pm 0.04$ & $2.01 \pm 0.04$ & $1.02 \pm 0.02$ \\
\hline $11.0-26.6$ & $2.568 \pm 0.004$ & $2.042 \pm 0.005$ & $1.90 \pm 0.01$ & $1.81 \pm 0.01$ & $1.22 \pm 0.01$ & $1.16 \pm 0.02$ & $1.62 \pm 0.03$ & $1.84 \pm 0.04$ & $0.97 \pm 0.02$ \\
\hline $26.6-65.3$ & $3.204 \pm 0.007$ & $0.683 \pm 0.002$ & $1.23 \pm 0.01$ & $1.22 \pm 0.01$ & $0.78 \pm 0.01$ & $1.13 \pm 0.02$ & $0.93 \pm 0.03$ & $1.09 \pm 0.04$ & $0.89 \pm 0.04$ \\
\hline \multicolumn{10}{|c|}{ Chandra deprojected } \\
\hline \multicolumn{10}{|c|}{$\begin{array}{l}\text { Deprojection method: dsdeproj } \\
\text { Model: vgadem }\end{array}$} \\
\hline $0-4.4$ & $1.187 \pm 0.001$ & $9.14 \pm 0.04$ & $1.09 \pm 0.01$ & $0.94 \pm 0.01$ & $0.81 \pm 0.02$ & $0.82 \pm 0.02$ & $1.40 \pm 0.14$ & $1.46 \pm 0.12$ & $1.34 \pm 0.11$ \\
\hline $4.4-11.0$ & $1.634 \pm 0.001$ & $3.918 \pm 0.003$ & $1.53 \pm 0.01$ & $1.52 \pm 0.01$ & $1.11 \pm 0.01$ & $0.94 \pm 0.02$ & $2.01 \pm 0.07$ & $2.01 \pm 0.06$ & $1.32 \pm 0.04$ \\
\hline $11.0-26.6$ & $2.573 \pm 0.006$ & $1.512 \pm 0.003$ & $1.94 \pm 0.01$ & $1.92 \pm 0.02$ & $1.29 \pm 0.01$ & $1.09 \pm 0.02$ & $1.82 \pm 0.04$ & $2.01 \pm 0.05$ & $1.04 \pm 0.03$ \\
\hline $26.6-65.3$ & $3.301 \pm 0.008$ & $0.573 \pm 0.001$ & $1.20 \pm 0.01$ & $1.21 \pm 0.01$ & $0.77 \pm 0.01$ & $1.09 \pm 0.02$ & $0.93 \pm 0.03$ & $1.09 \pm 0.04$ & $0.91 \pm 0.03$ \\
\hline \multicolumn{10}{|c|}{$\begin{array}{c}\text { Deprojection method: } d \text { sdeproj } \\
\text { Model: 2T vapec }\end{array}$} \\
\hline $0-4.4$ & $1.318 \pm 0.007$ & $10.82 \pm 0.11$ & $0.93 \pm 0.02$ & $0.81 \pm 0.02$ & $0.73 \pm 0.02$ & $0.68 \pm 0.03$ & $1.45 \pm 0.14$ & $1.47 \pm 0.11$ & $1.58 \pm 0.12$ \\
\hline $4.4-11.0$ & $1.600 \pm 0.001$ & $5.78 \pm 0.01$ & $1.40 \pm 0.01$ & $1.36 \pm 0.01$ & $0.99 \pm 0.01$ & $0.67 \pm 0.02$ & $1.91 \pm 0.07$ & $1.90 \pm 0.06$ & $1.36 \pm 0.04$ \\
\hline $11.0-26.6$ & $2.483 \pm 0.004$ & $2.096 \pm 0.003$ & $1.97 \pm 0.01$ & $1.92 \pm 0.01$ & $1.29 \pm 0.01$ & $1.09 \pm 0.02$ & $1.78 \pm 0.04$ & $1.98 \pm 0.06$ & $1.01 \pm 0.03$ \\
\hline $26.6-65.3$ & $3.196 \pm 0.005$ & $0.794 \pm 0.001$ & $1.23 \pm 0.01$ & $1.21 \pm 0.01$ & $0.77 \pm 0.01$ & $1.13 \pm 0.02$ & $0.94 \pm 0.03$ & $1.10 \pm 0.04$ & $0.89 \pm 0.04$ \\
\hline \multicolumn{10}{|c|}{$\begin{array}{c}\text { Deprojection method: projct } \\
\text { Model: vgadem }\end{array}$} \\
\hline $0-4.4$ & $1.173 \pm 0.002$ & $11.32 \pm 0.12$ & $1.104 \pm 0.003$ & $1.01 \pm 0.02$ & $0.87 \pm 0.03$ & $0.86 \pm 0.02$ & $1.07 \pm 0.23$ & $1.64 \pm 0.19$ & $1.30 \pm 0.20$ \\
\hline $4.4-11.0$ & $1.685 \pm 0.001$ & $4.22 \pm 0.03$ & $1.847 \pm 0.004$ & $1.74 \pm 0.01$ & $1.25 \pm 0.02$ & $0.90 \pm 0.02$ & $2.21 \pm 0.11$ & $2.29 \pm 0.11$ & $1.24 \pm 0.07$ \\
\hline $11.0-26.6$ & $2.517 \pm 0.003$ & $1.536 \pm 0.005$ & $2.319 \pm 0.005$ & $2.20 \pm 0.02$ & $1.53 \pm 0.02$ & $1.21 \pm 0.03$ & $2.23 \pm 0.08$ & $2.49 \pm 0.10$ & $1.05 \pm 0.05$ \\
\hline $26.6-65.3$ & $3.352 \pm 0.005$ & $0.513 \pm 0.001$ & $1.341 \pm 0.004$ & $1.34 \pm 0.01$ & $0.86 \pm 0.01$ & $1.16 \pm 0.02$ & $1.07 \pm 0.05$ & $1.28 \pm 0.07$ & $0.94 \pm 0.06$ \\
\hline \multicolumn{10}{|c|}{ XMM-Newton deprojected } \\
\hline \multicolumn{10}{|c|}{$\begin{array}{c}\text { Deprojection method: } d s d e p r o j \\
\text { Model: vgadem }\end{array}$} \\
\hline $0-4.4$ & $1.14 \pm 0.01$ & $7.38 \pm 0.12$ & $1.12 \pm 0.05$ & $0.92 \pm 0.05$ & $0.80 \pm 0.05$ & $0.76 \pm 0.06$ & $0.13 \pm 0.33$ & $1.82 \pm 0.30$ & $1.62 \pm 0.27$ \\
\hline $4.4-11.0$ & $1.75 \pm 0.01$ & $3.31 \pm 0.02$ & $2.06 \pm 0.05$ & $2.00 \pm 0.05$ & $1.29 \pm 0.04$ & $1.40 \pm 0.06$ & $1.75 \pm 0.11$ & $1.82 \pm 0.14$ & $0.88 \pm 0.07$ \\
\hline $11.0-26.6$ & $2.45 \pm 0.01$ & $1.445 \pm 0.004$ & $2.07 \pm 0.02$ & $1.88 \pm 0.03$ & $1.17 \pm 0.02$ & $1.14 \pm 0.04$ & $1.89 \pm 0.06$ & $1.70 \pm 0.09$ & $0.82 \pm 0.04$ \\
\hline $26.6-65.3$ & $3.33 \pm 0.01$ & $0.570 \pm 0.001$ & $1.49 \pm 0.01$ & $1.25 \pm 0.02$ & $0.63 \pm 0.02$ & $1.99 \pm 0.03$ & $0.94 \pm 0.04$ & $0.43 \pm 0.08$ & $0.29 \pm 0.05$ \\
\hline \multicolumn{10}{|c|}{$\begin{array}{c}\text { Deprojection method: projct } \\
\text { Model: } \text { vgadem }\end{array}$} \\
\hline $0-4.4$ & $1.62 \pm 0.27$ & $7.10 \pm 0.02$ & $1.19 \pm 0.03$ & $0.97 \pm 0.05$ & $0.79 \pm 0.08$ & $0.86 \pm 0.07$ & $0.00 \pm 0.40$ & $1.13 \pm 0.56$ & $0.95 \pm 0.47$ \\
\hline $4.4-11.0$ & $1.82 \pm 0.01$ & $3.49 \pm 0.02$ & $1.92 \pm 0.04$ & $1.71 \pm 0.04$ & $1.19 \pm 0.04$ & $1.15 \pm 0.06$ & $1.93 \pm 0.24$ & $1.81 \pm 0.24$ & $0.94 \pm 0.13$ \\
\hline $11.0-26.6$ & $2.35 \pm 0.01$ & $1.40 \pm 0.01$ & $1.96 \pm 0.02$ & $1.69 \pm 0.03$ & $1.08 \pm 0.04$ & $0.93 \pm 0.06$ & $1.90 \pm 0.16$ & $1.70 \pm 0.18$ & $0.89 \pm 0.09$ \\
\hline $26.6-65.3$ & $3.47 \pm 0.01$ & $0.584 \pm 0.001$ & $1.33 \pm 0.01$ & $1.07 \pm 0.03$ & $0.57 \pm 0.03$ & $1.60 \pm 0.05$ & $0.94 \pm 0.12$ & $0.48 \pm 0.15$ & $0.36 \pm 0.11$ \\
\hline \multicolumn{10}{|c|}{ Chandra deprojected narrow-band } \\
\hline \multicolumn{10}{|c|}{$\begin{array}{c}\text { Deprojection method: } d \text { sdeproj } \\
\text { Model: vgadem }\end{array}$} \\
\hline $0-4.4$ & - & - & $0.87 \pm 0.13$ & $1.09 \pm 0.02$ & $0.93 \pm 0.02$ & - & $1.33 \pm 0.18$ & $3.30 \pm 0.33$ & $3.07 \pm 0.31$ \\
\hline $4.4-11.0$ & - & - & $1.45 \pm 0.07$ & $1.77 \pm 0.01$ & $1.26 \pm 0.01$ & - & $2.49 \pm 0.09$ & $2.94 \pm 0.12$ & $1.93 \pm 0.08$ \\
\hline $11.0-26.6$ & - & - & $1.94 \pm 0.03$ & $1.95 \pm 0.01$ & $1.30 \pm 0.01$ & - & $2.02 \pm 0.06$ & $2.25 \pm 0.09$ & $1.17 \pm 0.04$ \\
\hline $26.6-65.3$ & - & - & $1.12 \pm 0.01$ & $1.15 \pm 0.01$ & $0.72 \pm 0.01$ & - & $1.13 \pm 0.04$ & $1.31 \pm 0.06$ & $1.09 \pm 0.05$ \\
\hline
\end{tabular}

Notes. We note that here we quote the mean temperature for the vgadem model and the higher temperature for the $2 \mathrm{~T}$ vapec model.

to get rid of these effects and investigate the drops as accurately as possible, it may be essential to deproject the spectra. As the first step, we deprojected the annular spectral regions using the dsdeproj tool developed by Russell et al. (2008). The method is based on the assumption of spherical symmetry, which is reasonable for the Centaurus cluster (see also Sect. 4.1). The deprojected spectra were analyzed in the same manner as described above for the projected profiles (see Sect. 3.1). The results for the radial distributions of temperature, total number density, the $\mathrm{Fe}, \mathrm{Si}, \mathrm{S}, \mathrm{Mg}, \mathrm{Ca}$ and $\mathrm{Ar}$ abundances, and the $\mathrm{Ar} / \mathrm{Fe}$ abundance ratio obtained using the two models are shown in Fig. 2 (red and blue data points) and Table 2 . In the $2 \mathrm{~T}$ vapec model, we find that only the innermost shell requires two components; the three outer shells can be reasonably fitted with a single-temperature component (vapec). This result is not surprising, as the apparent multi-temperature nature of the ICM due to projection effects is expected to be strongly reduced after deprojection.

Compared to their projected counterparts, the deprojected profiles modeled with the $2 \mathrm{~T}$ vapec and vgadem models show very similar trends. We note, however, that the deprojected Fe, $\mathrm{Si}, \mathrm{S}$ and $\mathrm{Mg}$ drops are somewhat more pronounced when using the $2 \mathrm{~T}$ vapec model. This may be explained by the fact that the shape of the unresolved Fe-L complex is rather sensitive to the assumed temperature structure. A change in the Fe abundance can, in turn, affect the abundance of the elements that are close to the Fe-L complex. The agreement found between our projected and deprojected profiles in the outermost annuli/shells is not surprising, as the latter are weakly affected by the deprojection methods. On the contrary, the largest differences are to be expected in the inner regions, where projection effects are more 

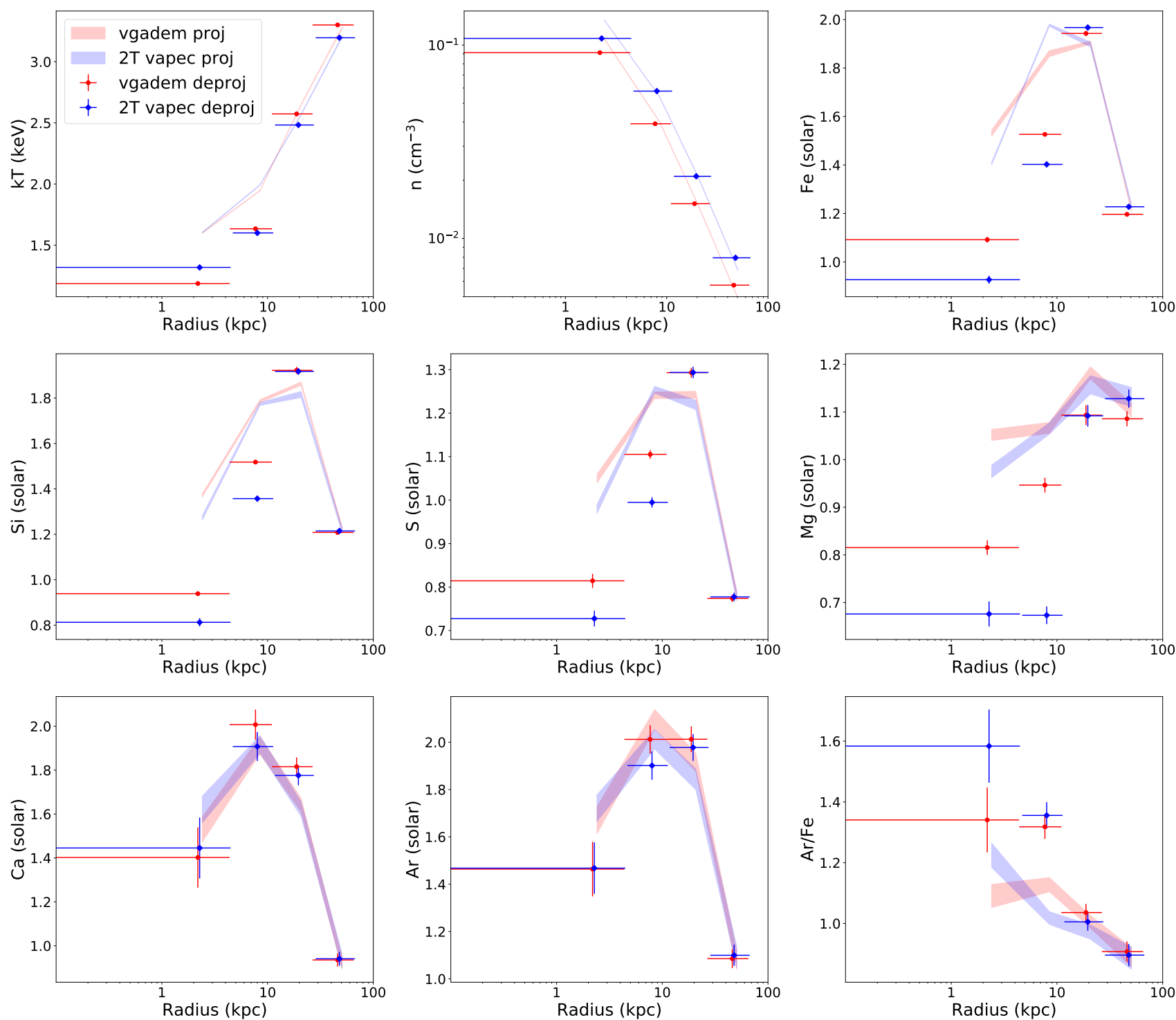

Fig. 2. A comparison of the projected (filled areas) and deprojected (data points) profiles of temperature, density, $\mathrm{Fe}, \mathrm{Si}, \mathrm{S}, \mathrm{Mg}, \mathrm{Ca}$ and $\mathrm{Ar}$ abundances and the $\mathrm{Ar} / \mathrm{Fe}$ abundance ratio, obtained using the vgadem (red) and $2 \mathrm{~T}$ vapec (blue) models. All errors are quoted at the $68.27 \%(1 \sigma)$ confidence level based on $\chi_{\min }^{2}$. The blue data points have been shifted slightly on the X-axis for clarity.

important. In fact, the Fe drop (spanning over the three innermost bins) appears much more significant in the deprojected profiles, in particular when the $2 \mathrm{~T}$ vapec model is assumed. In addition, the $\mathrm{Ar} / \mathrm{Fe}$ ratio also increases towards the center, with the same significance as in the projected profiles.

Since we have demonstrated that the choice of the particular multi-temperature model does not dramatically affect the shape of the metal abundance drops (in particular for Ar, i.e., our main element of interest), in the following we choose to use the vgadem model, both for its simplicity (one less free parameter than the $2 \mathrm{~T}$ vapec model) and realism (a continuous multitemperature distribution seems more plausible than only two distinct, independent gas phases). Moreover, the weaker $\mathrm{Ar} / \mathrm{Fe}$ gradient reported in Fig. 2 for the vgadem model makes our results more conservative.

\subsection{Deprojection methods}

To test the sensitivity of our results to different approaches to spectral deprojection, we compared our present approach (dsdeproj) with measurements obtained using the projct model in XSPEC. In the former method, for each shell the contributions of all the outer shells are subtracted and then each of the resulting deprojected spectra is fitted individually. The latter method involves forward fitting and the projected spectra of all the shells are fitted simultaneously to obtain the deprojected parameters. A comparison of the resulting profiles obtained using dsdeproj and projct is shown in Table 2 and Fig. 3 (red and blue data points).

The two deprojection methods yield rather similar metal abundance profile slopes. We note, however, that using projct brings the value of the maximum abundance for $\mathrm{Ca}$ to better agreement with the $\sim 10 \mathrm{kpc}$ extent of the $\mathrm{Fe}, \mathrm{Si}, \mathrm{S}$ and Mg drops. Furthermore, we find significantly higher abundances when using the projct method. While this source of uncertainties has no impact for the purpose of this study, we caution that it may be more critical if one wants to constrain the integrated abundances or abundance ratios of some specific elements in the ICM (e.g., Simionescu et al. 2019; Mernier et al. 2018b). Since projct involves a simultaneous fit of all the projected spectra, 

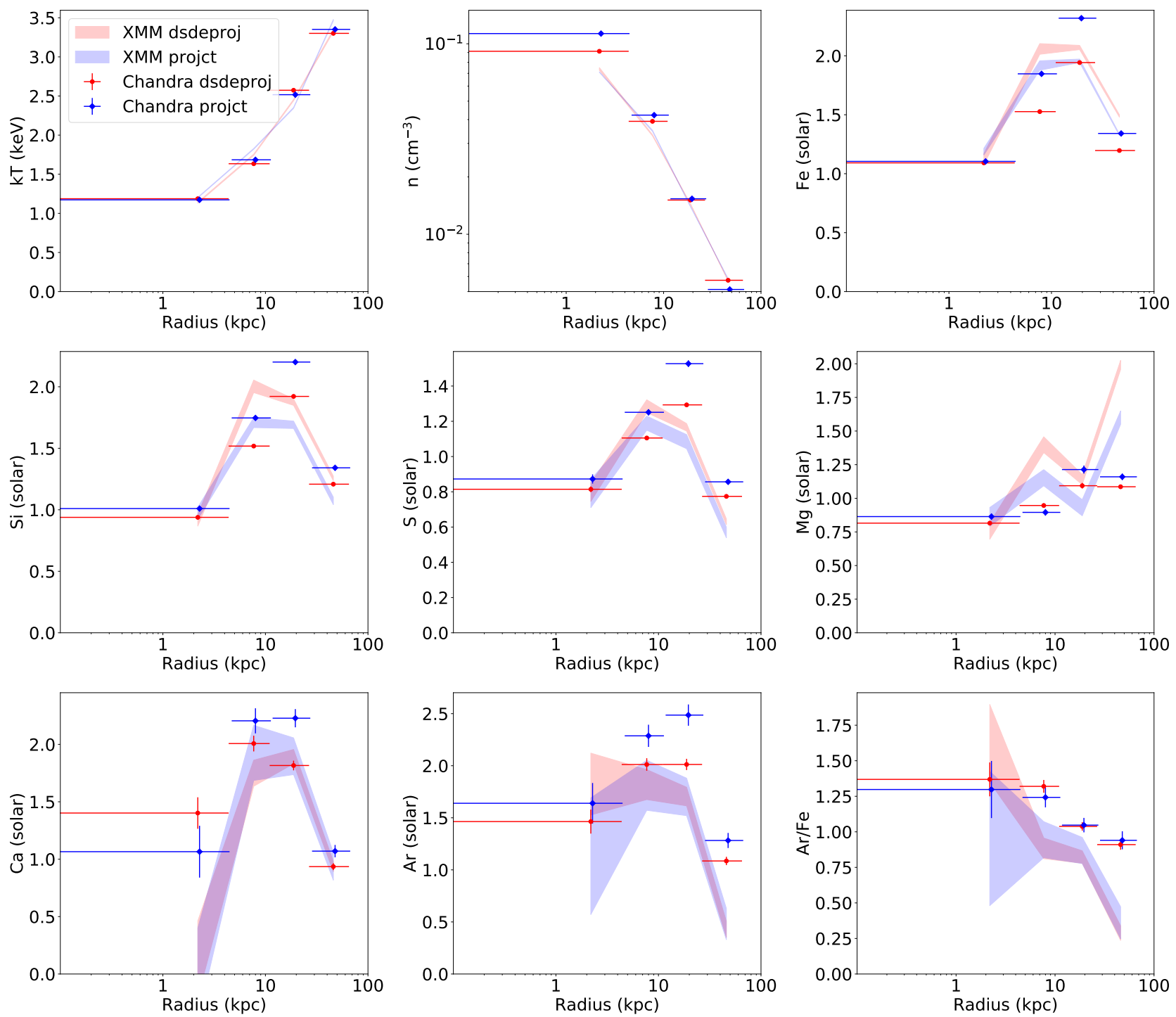

Fig. 3. A comparison of the Chandra (data points) and XMM-Newton (filled areas) temperature, density, Fe, Si, S, Mg, Ca and Ar abundances and the $\mathrm{Ar} / \mathrm{Fe}$ abundance ratio profiles, obtained using the vgadem model and deprojected using projct (blue) and dsdeproj (red). All errors are quoted at the $68.27 \%(1 \sigma)$ confidence level based on $\chi_{\min }^{2}$. The blue data points have been shifted slightly on the X-axis for clarity.

the derived uncertainties are higher for projct than for $d$ sdeproj. Therefore, although the magnitude of the drops in the best-fit values of the metal abundances are larger for projct than for $d s d e p r o j$, the significance of the drops is still higher for $d s d e$ $\operatorname{proj}(41 \sigma$ vs. $30 \sigma$ for $\mathrm{Fe})$. The inter-dependency of the shell parameters in projct is also known to produce potentially unstable measurements (Russell et al. 2008). However, the two deprojection techniques use different but equally valid approaches and the differences in the obtained results are likely to reflect the level of systematic uncertainties. For this reason, we show the results obtained with both methods. Because the implementation of $d$ sdeproj allows us to perform narrow band fits (see Sect. 3.5) more easily, we decided to use dsdproj as our reference deprojection method.

\subsection{Comparison with XMM-Newton}

In addition to comparing the results between dsdeproj and projct, Table 2 and Fig. 3 also compare the above Chandra/ACIS-S (density, temperature, metal abundance and $\mathrm{Ar} / \mathrm{Fe}$ ratio) profiles to the ones obtained with XMM-Newton/EPIC (red and blue filled areas).

We find that, globally, the XMM-Newton results are consistent (difference $<3 \sigma$ ) with their corresponding Chandra counterparts. The agreement is also in line with the analysis of Schellenberger et al. (2015), who found that the temperatures measured by ACIS and EPIC tend to converge when the gas is cooler. We note, however, a slight but significant tension between the two data sets in some specific cases - in particular in the second outermost shell when using projct. We speculate that these discrepancies may be due to a combination of remaining calibration issues and artifacts propagated when projct is used. Nevertheless, in both cases the deprojected EPIC spectra clearly reveal the presence of central drops for the $\mathrm{Fe}, \mathrm{Si}, \mathrm{S}, \mathrm{Mg}$ and $\mathrm{Ca}$ abundances.

Despite the formal consistency reported above, we note that the uncertainties of the XMM-Newton abundances of $\mathrm{Ar}$ and $\mathrm{Ca}$ are much larger than the uncertainties of the Chandra measurements. This point is discussed in more detail in Sect. 3.5. 

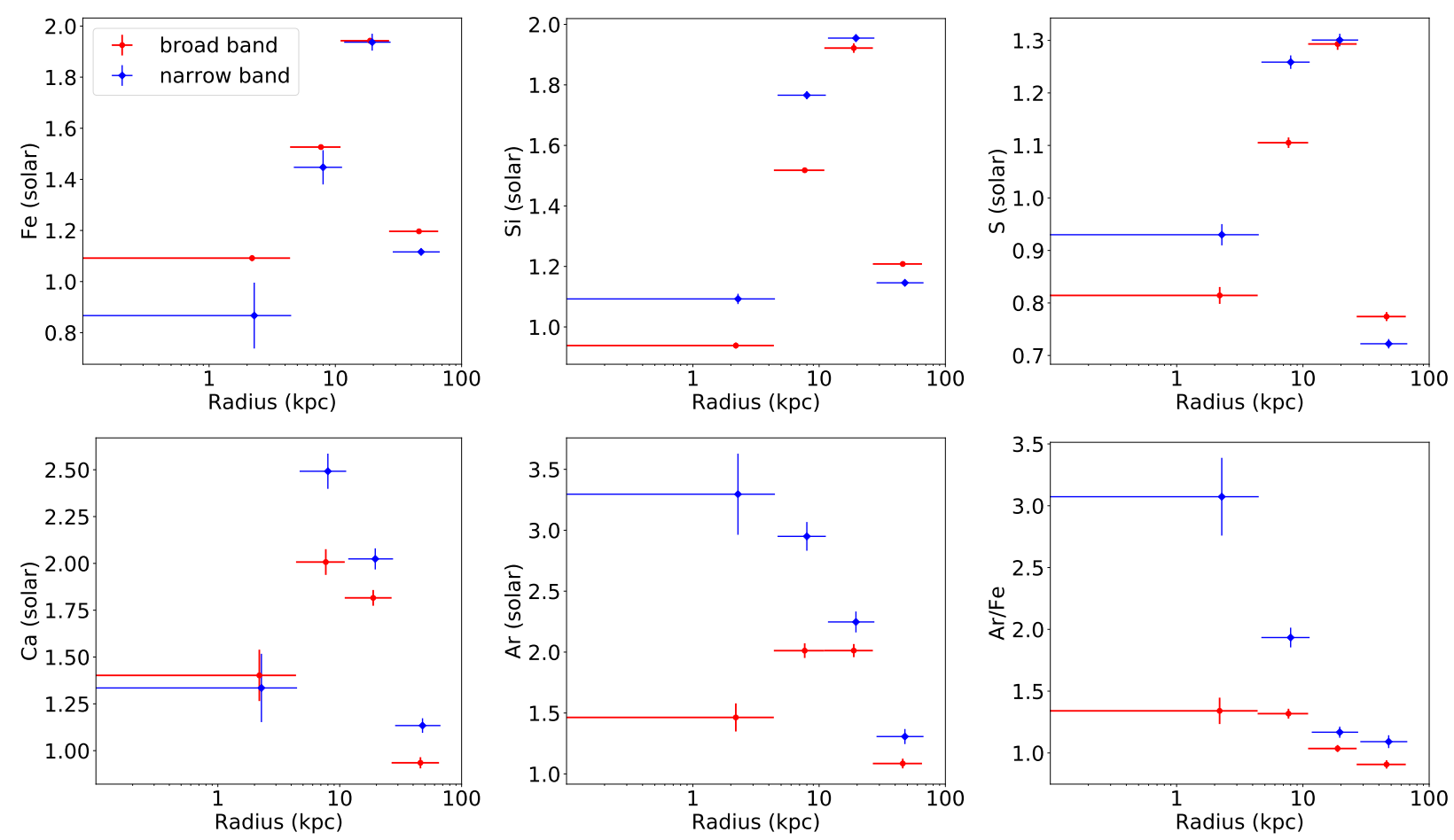

Fig. 4. Deprojected profiles of $\mathrm{Fe}, \mathrm{Si}, \mathrm{S}, \mathrm{Ca}$, and $\mathrm{Ar}$ abundances, and the $\mathrm{Ar} / \mathrm{Fe}$ (Fe taken from the broad-band fits) ratio, using broad- (red) and narrow-band (blue) fits, obtained using the vgadem model and the dsdeproj deprojection method. All errors are quoted at the $68.27 \%(1 \sigma)$ confidence level based on $\chi_{\min }^{2}$. The blue data points have been shifted slightly on the $\mathrm{X}$-axis for clarity.

\subsection{Broad-band versus narrow-band fits}

Whereas fitting the spectra using the full energy band (i.e., typically $\sim 0.5-7 \mathrm{keV}$ for Chandra ACIS) is the standard procedure for deriving the best-fit parameters, this approach may bias the metal abundance measurements. In fact, since the abundance of a given element is directly proportional to the equivalent width of its line (namely, the ratio between the total line flux and the continuum flux at the centroid energy of the line), incorrect determination of the continuum in the vicinity of the line would lead to incorrect measurement of the corresponding elemental abundance. Because no instrument is calibrated with infinite precision, some slight imperfections of the effective area may produce these local over- or underestimates of the continuum. In the case of very deep observations, that is, when statistical uncertainties become smaller than the above systematic effect, such a bias may become highly significant (e.g., Mernier et al. 2015; Simionescu et al. 2019).

A good way to minimize such biases is to refit the spectra between several local energy bands, each of them including the K-shell emission line(s) used to derive the abundance of a given element as well as part of the surrounding continuum. In these "narrow-band" fits (by contrast to the above "broadband" approach), the only parameters that were kept free were the (local) normalization and the abundance of the considered element. All the other parameters were fixed to the best-fit value of the broad-band fit. A comparison of the resulting narrow-band fit profiles with the ones obtained using broad-band fits (assuming a vgadem model and using dsdeproj) is shown in Table 2 and Fig. 4. We used the $1.5-2.8 \mathrm{keV}$ energy range for $\mathrm{Si}$ and $\mathrm{S}$, 2.9-3.5 keV for Ar, 3.3-4.3 keV for $\mathrm{Ca}$, and $5.0-7.0 \mathrm{keV}$ for $\mathrm{Fe}$.

The narrow-band $\mathrm{Fe}$ profile in Fig. 4 is consistent with the broad-band profile and also shows a drop in the innermost two bins (at $r \lesssim 10 \mathrm{kpc}$ ). The narrow-band fit results in larger statistical uncertainties on the Fe abundance, because it only includes the Fe-K lines and excludes the Fe-L complex. Despite the larger error bars on the measurements, the Fe drop in the narrow-band case is significant at the $>8 \sigma$ level. Similarly to the broad-band profiles, the narrow-band profiles of $\mathrm{Si}, \mathrm{S}$, and $\mathrm{Ca}$ also show significant central drops (at the $\sim 41,16$, and $6 \sigma$ levels, respectively). Importantly, the narrow-band abundances of all the elements other than $\mathrm{Fe}$ are significantly higher than their broad-band values, especially in the innermost two bins. Thisindicates an overestimation of the continuum emission in the $\sim 1.5-5 \mathrm{keV}$ range when the spectra are fitted over the entire $0.5-7 \mathrm{keV}$ band.

Interestingly, the $\mathrm{Ar}$ abundance profile determined using narrow-band fits (Fig. 4, bottom left), differs even more from the corresponding broad-band results than the other profiles reported above. As reported in Sect. 3.2, the broad-band Ar profile shows a $2 \sigma$ hint of a drop in the innermost bin, while the highest Ar value peaks between 4 and $27 \mathrm{kpc}$. However, the continuum around the Ar line in the innermost bin was found to be slightly overestimated. We also confirm the overestimation of the continuum using projct. After applying the narrow-band fit correction, the centrally decreasing behavior of Ar completely disappears and becomes either flat or increasing towards the center. Perhaps even more importantly, the central increase of the $\mathrm{Ar} / \mathrm{Fe}$ ratio $^{3}$ reported earlier is now strongly enhanced ( $>3 \sigma$ comparing the innermost two bins; $>6 \sigma$ when comparing the innermost and outermost bins; Fig. 4, bottom right).

\footnotetext{
3 In the Ar/Fe profile shown in Fig. 4, we choose to adopt the Fe values from their broad-band measurements because the Fe-L complex provides additional constraints while not deviating significantly from the narrow-band values. We note that, when adopting the narrow-band Fe values in the $\mathrm{Ar} / \mathrm{Fe}$ profile, the central increase remains firmly detected.
} 


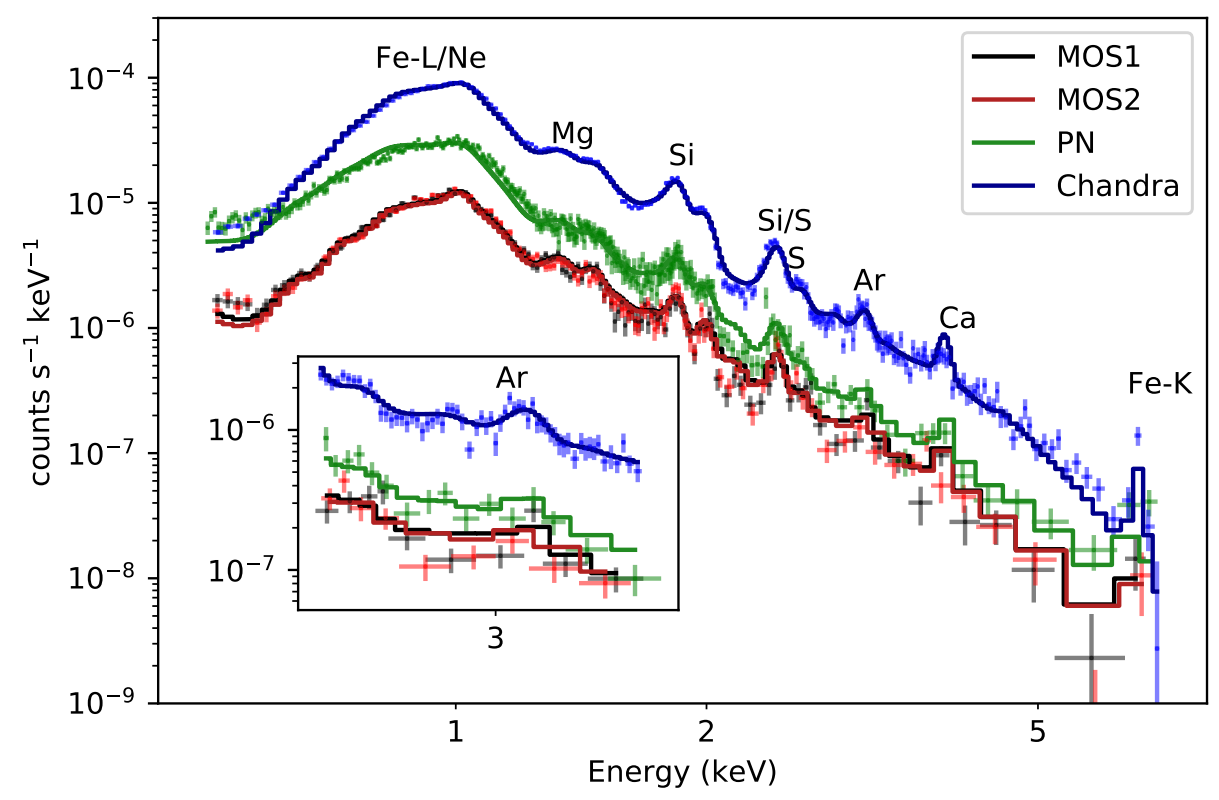

Fig. 5. A comparison of the deprojected Chandra (blue) and the XMM-Newton MOS1 (black), MOS2 (red), and PN (green) spectra of the innermost shell; the most important emission lines have been marked. The Chandra spectrum has been scaled up by a factor of five for viewing purposes. The inset shows the zoomed-in spectra around the Ar line. It can be seen that a slight over/underestimation of the continuum in the broad-band fit can lead to an under/overestimation of the abundances, especially for the weak lines. It is also clearly seen that the XMM-Newton spectra around the high-energy lines ( $\mathrm{Ar}, \mathrm{Ca}$ and $\mathrm{Fe}-\mathrm{K}$ ) are very noisy and are not as resolved as in the Chandra spectrum and therefore the XMM-Newton spectra are not found suitable for the narrow-band analyses.

Given the rather deep ( $\sim 150 \mathrm{ks})$ XMM-Newton observations that are available, and motivated by our previous comparison in Sect. 3.4, we also attempted to perform narrow-band fits in the deprojected XMM-Newton spectra. Regrettably, very few additional constraints could be obtained for $\mathrm{Ar}, \mathrm{Ca}$, and $\mathrm{Fe}$ using that approach (see also Sect. 3.4). In fact, the limited number of counts for each of the EPIC instruments, coupled with the spectral grouping requirements of $d s d e p r o j$, make narrow-band fits of their deprojected spectra around the Ar line very difficult. This is illustrated further in Fig. 5, where beyond $\sim 2 \mathrm{keV}$ the combined deprojected spectra of ACIS-S is better resolved than the combined deprojected spectra of EPIC MOS 1, MOS 2, and pn. In theory, better constraints from the XMM-Newton data would be possible if the three EPIC spectra were stacked instead of being fitted simultaneously; however this method is not advised as it may introduce unexpected uncertainties from the combined response and effective area.

For this reason, we choose to rely primarily on the deeper Chandra observations, as they allow us to keep all the systematic effects mentioned above under control.

\section{Discussion}

\subsection{Systematic uncertainties and limitations of the analysis}

As demonstrated throughout this paper, a careful analysis of the spectra is extremely important for accurately measuring metal abundances and their distribution. Indeed, if the spectra are fitted using overly simplistic assumptions, the subsequent incorrect determination of the metal abundance profiles can easily produce artificial trends or, on the contrary, wipe out actual features. From our analyses, we report that the following factors can significantly affect the measurements of the metal abundance drops:

Temperature distribution effects. An incorrect modeling of the thermal structure of the ICM can simultaneously affect the continuum emission and the modeling of the mostly unre- solved spectral lines. This naturally results in incorrect abundance determinations. One of the best examples is the Fe bias which leads to an underestimated Fe abundance when using a single-temperature model to describe a multi-temperature plasma (Buote 2000, see also Mernier et al. 2018a). As shown by Werner et al. (2006), this Fe bias can easily create artificial Fe drops in some cases. In addition, our present analysis shows that even the choice of the multi-temperature model can alter the shape of the abundance drops (see e.g., the Fe drop in Fig. 2). This effect is nevertheless less important than the Fe bias.

Projection effects. Projection along the line of sight, especially in systems with strong temperature gradients, can lead to the mixing of the emission of the cooler gas in the cluster center with the emission of the hotter ICM at larger radii. The projected view of the central low-metallicity gas overlapping with the higher-metallicity outer layers will essentially result in overestimation of the abundances in the central regions. These projection effects can explain the relatively weaker central drops seen in the projected as compared to the deprojected profiles (Fig. 2). As shown in Fig. 3, different deprojection techniques result in profiles with consistent shapes, but may add some extra uncertainties to the measurements of the absolute abundance values.

Continuum effects. We found that the abundances of weak lines can be significantly over-/underestimated, even by a slightly incorrect modeling of the local continuum level (see Sect. 3.5 and Fig. 4). As explained in Sect. 3.5, the best way to deal with this effect is to fit these lines individually, within narrow energy bands. In the case of Ar, this effect is significant and clearly affects the interpretations and conclusions of the present study (see also Sect. 4.2). Therefore, we caution that narrow-band fits should be systematically considered when one aims to measure accurate abundances from very deep observations of X-ray bright clusters. In our case, the $\mathrm{Fe}$ abundances measured with only the K-shell transitions are formally consistent with those measured using the full energy band. We note, 
however, that in the central cooler regions of the ICM, Fe abundances are often derived from the Fe-L complex, which is very sensitive to the modeling uncertainties of the temperature structure of the plasma. A possible mismatch between broad-band and narrow-band derived Fe abundances in other systems may thus reveal a more complicated temperature structure than initially assumed.

Instrumental calibration, data quality, and detector PSF. Because no X-ray mission is perfectly calibrated, comparing measurements done with the same analysis methods using two or more instruments is strongly recommended for very deep data. For instance, it is well known that XMM-Newton and Chandra do not measure the same X-ray temperatures (Schellenberger et al. 2015). In the Centaurus cluster, we show that these two telescopes sometimes provide slight but significant differences in metal abundances as well. Finally, although these effects should be limited in our analysis, we note that the spatial resolution of the instrument can also play an important role as a broader PSF can smooth out gradients.

Considering the above factors, we argue that the deprojected narrow-band analysis presented in Sect. 3.5, fitted with the Gaussian-shaped multi-temperature model vgadem, and based on the Chandra data (i.e., taking advantage of its unprecedented PSF of $\sim 0.5^{\prime \prime}$ ), provides the most reliable measurements to date of the central abundance drops - especially when emission lines are weak. We note however, that some limitations still remain and could be resolved only with future missions and instruments:

- As the ICM becomes more multi-temperature when approaching the cluster center, it is not clear how the metal abundances should be associated with the different thermal components. With the current spectral resolution of CCDs, assuming different abundances for two or more individual (single-temperature) components would considerably increase the degeneracy between parameters. Determining how complex abundance distributions would affect the central drops requires comprehensive high-resolution simulations that need to be analyzed using mock spectra (for an example of simulated clusters investigated via mock $X M M$ Newton data, see e.g., Rasia et al. 2008).

- While in our analysis we have tried two simplified assumptions to model the multi-temperature plasma (i.e., the $2 \mathrm{~T}$ vapec and the vgadem models), the actual emission might be different from both these assumptions, and might even have an asymmetric emission measure distribution. Determining the real temperature distribution in the ICM is far from trivial - even when the spectral resolution is considerably improved (Hitomi Collaboration 2018) - and probably requires a very good spatial resolution as well.

- All deprojection methods rely on the assumption of spherical symmetry. Based on the relatively regular morphology of the $\mathrm{X}$-ray emission of the Centaurus cluster, this seems to be a reasonable assumption and the observed modest departures from spherical symmetry are unlikely to affect our conclusions.

More generally, the careful successive checks performed in the present study have shown that the presence of drops in metal abundance is strongly established. It is also important to note that, since for these very deep data sets the systematic uncertainties dominate over the statistical ones, adding more Chan$d r a$ and/or XMM-Newton exposures in the future is not expected to significantly improve the accuracy of the present measurements. While observations with future instruments will be absolutely necessary for this purpose, we speculate that all the limitations discussed above would somewhat affect the magni- tude of the metal abundance drops, but are not expected to significantly change our conclusions.

\subsection{The origin of the metal abundance drops}

Using the best data that are available to date for the Centaurus cluster, our comprehensive analysis confirms the central drops seen in the abundances of the reactive elements $\mathrm{Fe}, \mathrm{Si}, \mathrm{S}, \mathrm{Mg}$ and $\mathrm{Ca}$, with a much greater significance than obtained previously (see Sanders \& Fabian 2002, 2006a; Panagoulia et al. 2013; Sanders et al. 2016). After adopting our preferred analysis approach (i.e., vgadem multi-temperature model, ACIS-S spectra deprojected using dsdeproj, and abundances fitted individually within a narrow-band), we have checked carefully that these drops do not disappear when assuming a departure from these assumptions. Using narrow-band fits, the $\mathrm{Fe}, \mathrm{Si}, \mathrm{S}$ and $\mathrm{Mg}$ drops are seen inside a region of $\sim 10 \mathrm{kpc}$ from the center. For these elements, the spatial extent of the drops is broadly consistent with Panagoulia et al. (2013). It is also interesting to note that the inner region, where the bulk of the central metals is missing, overlaps remarkably well with the dust emission region seen by Mittal et al. (2011) (see also Fig. 1). However, the Ar abundance is fully consistent with no drop at the center. Instead, the deprojected narrow-band fits (Fig. 4) show a monotonic centrally increasing trend for Ar. More importantly, even taking all the above systematic uncertainties into account, we always see a significant increase in the $\mathrm{Ar} / \mathrm{Fe}$ ratio towards the center (at least $>2 \sigma$ from $\sim 60 \mathrm{kpc}$ to $\sim 2 \mathrm{kpc}$ )

As discussed in Sect. 1, the radial distribution of Ar across the location of the Fe drop constitutes a crucial prediction of the dust-depletion model in the central ICM, as proposed by Panagoulia et al. (2013, 2015). Together with the observed significant central drops in the abundances of reactive elements Fe, $\mathrm{Si}, \mathrm{S}, \mathrm{Mg}$ and $\mathrm{Ca}$, the peaked profile seen in the central region for a noble gas like $\mathrm{Ar}$, and the central increase of the $\mathrm{Ar} / \mathrm{Fe}$ abundance ratio, strongly suggest that a significant fraction of the cooled reactive metals is indeed getting deposited into the dust grains present in the central regions of the Centaurus cluster.

Qualitatively, our observations seem thus to indicate that metal abundance drops are real and form via the above channel. As explained in Sect. 1, the drops may also become more pronounced due to mechanical feedback from the central AGN which can uplift the metal-rich dust grains out of the central regions towards outer hotter regions, where they get re-heated and start emitting in the X-ray band again.

Another open question is the mismatch in the spatial extent of the abundance drops (e.g., Fig. 4) and the spatial extent of the dust emission observed in the far-infrared (Fig. 1). While the metal abundance drops typically extend out to $\sim 10 \mathrm{kpc}$ (possibly somewhat further; e.g., Panagoulia et al. 2013), the dust emission is only seen within $\sim 4 \mathrm{kpc}$, that is, it is entirely contained within our innermost spectral extraction region. It is possible, however, that a significant amount of dust resides outside $\sim 4 \mathrm{kpc}$, where its associated infrared emission is too weak to be firmly detected with Herschel and Spitzer. Another possibility is that most of the dust uplifted by the AGN activity to radii between $\sim 10 \mathrm{kpc}$ and $\sim 27 \mathrm{kpc}$ is destroyed by the interaction with the ICM. In that case, the metal abundance peak, observed in the third shell, is due to the excess of metals that have freshly returned to the ICM.

Finally, we note that the presence of a central Ar drop would not necessarily rule out the existence of a depletion of the central ICM-phase metals and their incorporation into dust grains. As long as the $\mathrm{Ar} / \mathrm{Fe}$ abundance ratio is constantly increasing 
towards the cluster core, dust could play a significant role in lowering the central ICM abundances.

\section{Summary}

In this study, we performed detailed, projected and deprojected spectral analyses of very deep Chandra and XMM-Newton Xray observations of the Centaurus cluster. We also investigated the various factors that could bias the metal abundance measurements. These factors include an incorrect modeling of the temperature distribution of the plasma, projection along the line of sight, uncertainties in the modeling of the local continuum (especially when weak emission lines are fitted over a broad energy band), poor data quality, as well as detector PSF and calibration.

When adopting our most conservative approach, taking most of these systematic effects into account, we confirm the central drops seen in the abundance profiles of the reactive elements such as, $\mathrm{Fe}, \mathrm{Si}, \mathrm{S}, \mathrm{Mg}$ and $\mathrm{Ca}$, with a $>6 \sigma$ confidence. For $\mathrm{Fe}, \mathrm{Si}, \mathrm{S}$, and $\mathrm{Mg}$, the drops are seen at $r \sim 10 \mathrm{kpc}$, while for $\mathrm{Ca}$, a significant drop is seen only at $r \sim 4 \mathrm{kpc}$. The region of these metal abundance drops therefore broadly coincides with the central dust emission. Additionally, we find a centrally increasing/flattening trend in the abundance of the noble gas $\mathrm{Ar}$, further confirmed by a significant increase in the $\mathrm{Ar} / \mathrm{Fe}$ ratio towards the innermost region of the cluster. These results constitute strong observational support in favor of the scenario proposed by Panagoulia et al. $(2013,2015)$ in which the drops in central metal abundance are due to the incorporation of a significant fraction of the cooling reactive elements into dust grains, leading to their disappearance from the X-ray band.

Acknowledgements. This work was supported by the Lendület LP2016-11 grant awarded by the Hungarian Academy of Sciences. SRON is supported financially by NWO, the Netherlands Organization for Scientific Research. This research has made use of software provided by the Chandra X-ray Centre (CXC) in the application packages CIAO, CHIPS and Sherpa. The scientific results reported in this article are based to a significant degree on data, software and web tools obtained from the High Energy Astrophysics Science Archive Research Center (HEASARC), a service of the Astrophysics Science Division at NASA/GSFC and of the Smithsonian Astrophysical Observatory's High Energy Astrophysics Division. The scientific results reported in this article are based on observations made by the Chandra X-ray Observatory. This work is based on observations obtained with XMM-Newton, an ESA science mission with instruments and contributions directly funded by ESA member states and the USA (NASA).

\section{References}

Arnaud, K. A. 1996, in Astronomical Data Analysis Software and Systems V, eds. G. H. Jacoby, \& J. Barnes, ASP Conf. Ser., 101, 17

Biffi, V., Planelles, S., Borgani, S., et al. 2017, MNRAS, 468, 531
Biffi, V., Planelles, S., Borgani, S., et al. 2018a, MNRAS, 476, 2689 Biffi, V., Mernier, F., \& Medvedev, P. 2018b, Space Sci. Rev., 214, 123 Böhringer, H., \& Werner, N. 2010, A\&ARv, 18, 127

Böhringer, H., Matsushita, K., Churazov, E., Finoguenov, A., \& Ikebe, Y. 2004, A\&A, 416, L21

Buote, D. A. 2000, MNRAS, 311, 176

Churazov, E., Forman, W., Jones, C., \& Böhringer, H. 2003, ApJ, 590, 225

Churazov, E., Forman, W., Jones, C., Sunyaev, R., \& Böhringer, H. 2004, MNRAS, 347, 29

De Grandi, S., Ettori, S., Longhetti, M., \& Molendi, S. 2004, A\&A, 419, 7

Fabian, A. C., Walker, S. A., Russell, H. R., et al. 2016, MNRAS, 461, 922

Fruscione, A., McDowell, J. C., Allen, G. E., et al. 2006, SPIE Conf. Ser., 6270, $62701 \mathrm{~V}$

Gendron-Marsolais, M., Kraft, R. P., Bogdan, A., et al. 2017, ApJ, 848, 26

Grevesse, N., \& Sauval, A. J. 1998, Space Sci. Rev., 85, 161

Hamer, S. L., Fabian, A. C., Russell, H. R., et al. 2019, MNRAS, 483, 4984

Hitomi Collaboration (Aharonian, F., et al.) 2018, PASJ, 70, 11

Hudson, D. S., Mittal, R., Reiprich, T. H., et al. 2010, A\&A, 513, A37

Irwin, J. A., Athey, A. E., \& Bregman, J. N. 2003, ApJ, 587, 356

Johnstone, R. M., Allen, S. W., Fabian, A. C., \& Sanders, J. S. 2002, MNRAS, 336, 299

Kaneda, H., Onaka, T., \& Sakon, I. 2005, ApJ, 632, L83

Kaneda, H., Onaka, T., Kitayama, T., Okada, Y., \& Sakon, I. 2007, PASJ, 59, 107

Lakhchaura, K., Werner, N., Sun, M., et al. 2018, MNRAS, 481, 4472

Lovisari, L., \& Reiprich, T. 2019, MNRAS, 483, 540

Lumb, D. H., Warwick, R. S., Page, M., \& De Luca, A. 2002, A\&A, 389, 93

Mernier, F., de Plaa, J., Lovisari, L., et al. 2015, A\&A, 575, A37

Mernier, F., de Plaa, J., Kaastra, J. S., et al. 2017, A\&A, 603, A80

Mernier, F., Biffi, V., Yamaguchi, H., et al. 2018a, Space Sci. Rev., 214, 129

Mernier, F., Werner, N., de Plaa, J., et al. 2018b, MNRAS, 480, L95

Mittal, R., O'Dea, C. P., Ferland, G., et al. 2011, MNRAS, 418, 2386

Molendi, S., \& Pizzolato, F. 2001, ApJ, 560, 194

Panagoulia, E. K., Fabian, A. C., \& Sanders, J. S. 2013, MNRAS, 433, 3290

Panagoulia, E. K., Sanders, J. S., \& Fabian, A. C. 2015, MNRAS, 447, 417

Rafferty, D. A., Bîrzan, L., Nulsen, P. E. J., et al. 2013, MNRAS, 428, 58

Rasia, E., Mazzotta, P., Bourdin, H., et al. 2008, ApJ, 674, 728

Rasmussen, J., \& Ponman, T. J. 2007, MNRAS, 380, 1554

Russell, H. R., Sanders, J. S., \& Fabian, A. C. 2008, MNRAS, 390, 1207

Sanders, J. S., \& Fabian, A. C. 2002, MNRAS, 331, 273

Sanders, J. S., \& Fabian, A. C. 2006a, MNRAS, 371, 1483

Sanders, J. S., \& Fabian, A. C. 2006b, MNRAS, 370, 63

Sanders, J. S., Fabian, A. C., Taylor, G. B., et al. 2016, MNRAS, 457, 82

Schellenberger, G., Reiprich, T. H., Lovisari, L., Nevalainen, J., \& David, L. 2015, A\&A, 575, A30

Simionescu, A., Werner, N., Böhringer, H., et al. 2009, A\&A, 493, 409

Simionescu, A., Werner, N., Urban, O., et al. 2015, ApJ, 811, L25

Simionescu, A., Nakashima, S., Yamaguchi, H., et al. 2019, MNRAS, 483, 1701

Truong, N., Rasia, E., Biffi, V., et al. 2019, MNRAS, 484, 2896

Urban, O., Werner, N., Allen, S. W., Simionescu, A., \& Mantz, A. 2017, MNRAS, 470, 4583

Werner, N., de Plaa, J., Kaastra, J. S., et al. 2006, A\&A, 449, 475

Werner, N., Urban, O., Simionescu, A., \& Allen, S. W. 2013, Nature, 502, 656

Werner, N., McNamara, B. R., Churazov, E., \& Scannapieco, E. 2019, Space Sci. Rev., 215, 5 